\title{
Recurrent somatic JAK-STAT pathway variants within a RUNX1-mutated pedigree
}

\author{
Kiran Tawana ${ }^{\star}, 1$, Jun Wang ${ }^{2}$, Péter A Király ${ }^{3}$, Krisztián Kállay ${ }^{4}$, Gábor Benyó ${ }^{4}$, Marianna Zombori ${ }^{5}$, \\ Judit Csomor ${ }^{3}$, Ahad Al Seraihi ${ }^{1}$, Ana Rio-Machin ${ }^{1}$, András Matolcsy ${ }^{3}$, Claude Chelala ${ }^{2}$, Jamie Cavenagh ${ }^{1}$, \\ Jude Fitzgibbon ${ }^{1}$ and Csaba Bödör ${ }^{\star, 3}$
}

Germline variants within the transcription factor RUNX1 are associated with familial platelet disorder and acute leukemia in over $40 \%$ of carriers. At present, the somatic events triggering leukemic transformation appear heterogeneous and profiles of leukemia initiation across family members are poorly defined. We report a new RUNX1 family where three sisters harboring a germline nonsense RUNX1 variant, c.601C $>\mathrm{T}\left(\mathrm{p} .\left(\mathrm{Arg} 201^{*}\right)\right.$ ), developed acute myelomonocytic leukemia (AML) at 5 years of age. Whole-exome sequencing of tumor samples revealed all three siblings independently acquired variants within the JAK-STAT pathway, specifically targeting JAK2 and SH2B3 (a negative regulator of JAK2), while also sharing the 46/1 haplotype linked with sporadic JAK2-positive myeloproliferative neoplasms. In-depth chromosomal characterization of tumors revealed acquired copy number gains and uniparental disomy amplifying RUNX1, JAK2 and SH2B3 variants, highlighting the significance of cooperation between these disrupted pathways. One sibling, presenting with myelodysplasia at 14 years, had no evidence of clonal or subclonal JAK2 or SH2B3 variants, suggesting the latter were specifically associated with leukemic transformation in her sisters. Collectively, the clinical and molecular homogeneity across these three young siblings provides the first notable example of convergent AML evolution in a RUNX1 pedigree, with the recurrent acquisition of JAK-STAT pathway variants giving rise to high-risk AML, characterized by chemotherapy resistance and relapse.

European Journal of Human Genetics (2017) 25, 1020-1024; doi:10.1038/ejhg.2017.80; published online 17 May 2017

\section{INTRODUCTION}

Acquired RUNX1 variants occur in $\sim 10 \%$ of sporadic acute myelomonocytic leukemia (AML) cases and the clinico-pathologic associations within this subgroup of disease have been comprehensively defined. ${ }^{1}$ Inherited or germline RUNX1 variants are comparatively rare and typically cluster in the highly conserved runt homology domain, predisposing to familial platelet disorder and myelodysplasia (MDS) or acute leukemia. ${ }^{2}$ Leukemic transformation occurs in over $40 \%$ of variant carriers, coinciding with a heterogeneous profile of somatic genetic lesions, although aberrations of the second RUNX1 allele or amplification of the germline variant are frequent. ${ }^{3,4}$ Notably, $C D C 25 C$ variants were reported as a recurrent event in a Japanese RUNX1-mutated cohort, ${ }^{5}$ however these results were not replicated in the US and European studies, ${ }^{3,6,7}$ suggesting ethnicity may influence somatic variant acquisition. Exploring this notion further, we considered whether family members may develop similar patterns of disease, due to their common genetic background. Using comprehensive clinical and genetic profiling of multiple siblings with MDS/AML, we report striking phenotypic and molecular convergence of AML within a new Hungarian RUNX1-mutated family.

\section{DESIGN AND METHODS}

We used the SureSelect All Exon V5 kit (Agilent, Santa Clara, CA, USA) to perform whole-exome sequencing (WES) on bone marrow (BM) tumor DNA from siblings (II.1-II.4) and peripheral blood (PB) DNA from both healthy parents (I.1 and I.2), the latter providing a germline reference. Enriched exome libraries were sequenced to an average depth of $\times 96$ (Supplementary Table S1) using the HiSeq 2000 (Illumina, San Diego, CA, USA) with additional analysis described in Supplementary Data.

An established pipeline incorporating MuTect (single-nucleotide variants, SNVs) and GATK (indels) was employed for variant calling in tumor samples. Analysis of chromosomal loss of heterozygosity, including copy number aberrations (CNAs) and acquired uniparental disomy (aUPD) is detailed in the Supplementary Data. Sequence variants and CNAs were verified using Sanger sequencing and multiplex ligation-dependent probe amplification (MRC Holland, Amsterdam, the Netherlands, SALSA X060-X2), respectively. The WES data have been deposited in the European Genome-Phenome Archive under the accession number EGAS00001001862. Deep sequencing of JAK2 exon 14 and all coding exons of SH2B3 was performed using the AmpliSeq approach on an Ion Torrent (Life Technologies, Carlsbad, CA, USA) instrument. The reference sequences used for variant annotations are listed in the Supplementary Data.

\section{RESULTS}

A nonsense RUNX1 variant, c.601C > T (p. $\left.\left(\operatorname{Arg} 201^{\star}\right)\right)$, was detected in all four siblings and their mother, (Figures $1 \mathrm{a}$ and b; Supplementary Table S2). The first affected family members were dizygotic twins (II.1 and II.2) who presented in 2002 at 5 years of age, within a period of 14 days. They both had hepatosplenomegaly and monocytosis, with BM morphology revealing AML (FAB AML M4). After 6 years, sibling

${ }^{1}$ Centre for Haemato-Oncology, Barts Cancer Institute, Queen Mary University of London, London, UK; ${ }^{2}$ Bioinformatics Unit, Barts Cancer Institute, Queen Mary University of London, London, UK; ${ }^{3} 1$ st Department of Pathology and Experimental Cancer Research, MTA-SE Lendulet Molecular Oncohematology Research Group, Semmelweis University, Budapest, Hungary; ${ }^{4}$ Pediatric Haematology and Stem Cell Transplantation Unit, United St. István and St. László Hospital, Budapest, Hungary; ${ }^{5}$ Department of OncoHaematology, Heim Pál Children's Hospital, Budapest, Hungary

*Correspondence: Dr K Tawana or Dr C Bödör, 1st Department of Pathology and Experimental Cancer Research, MTA-SE Lendulet Molecular Oncohematology Research Group, Semmelweis University, Ulloi ut 26, Budapest 1085, Hungary. Tel: +36 12157300 ext: 54462; Fax: +36 13171074 . E-mail: bodor.csaba1@med.semmelweis-univ.hu or k.tawana@qmul.ac.uk

Received 17 February 2017; revised 2 April 2017; accepted 13 April 2017; published online 17 May 2017 
a

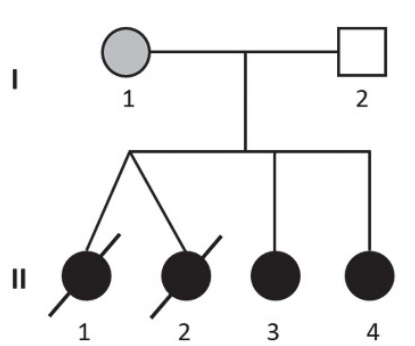

b

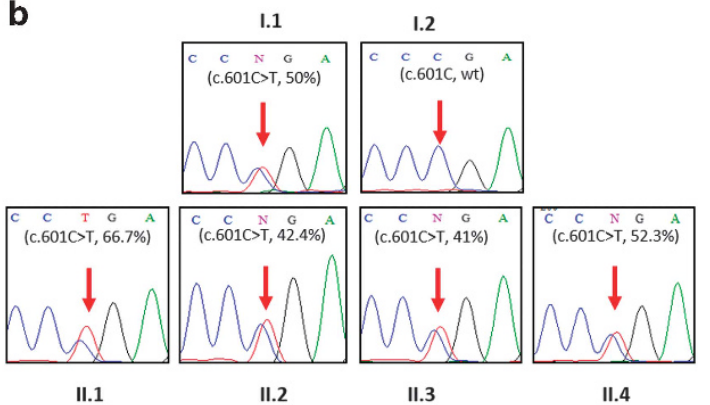

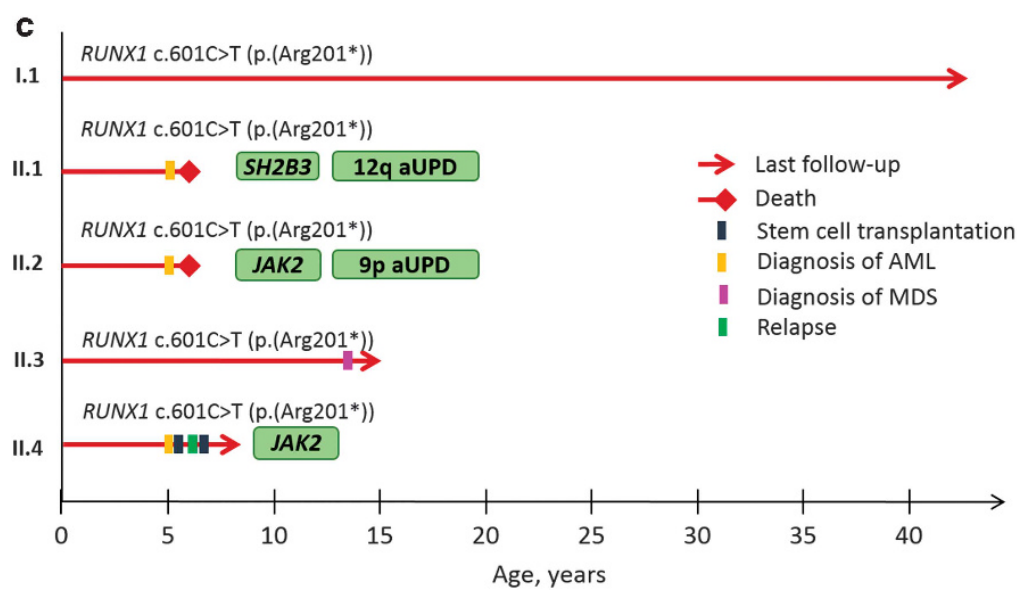

Figure 1 Profile of a novel RUNX1-mutated pedigree. (a) Novel RUNX1 pedigree showing affected individuals (black shading) and asymptomatic carriage of germline variant (gray shading). (b) RUNX1 c.601C > T (p.(Arg201*)) variant identified in four siblings and their mother, the corresponding WES VAFs are shown. (c) Clinical timeline of the pedigree showing siblings II.1, II.2 and II.4 presenting with AML at 5 years of age. All three siblings with AML acquired variants upregulating JAK-STAT signaling, while sibling 1 I.3 remains under expectant follow-up with MDS. (RUNX1 reference sequences: ENSG00000159216 / ENST00000300305).

II.4 presented with thrombocytopenia aged 1 year, followed by AML (FAB AML M4) at 5 years of age. In contrast, sibling II.3 developed mild anemia and thrombocytopenia, and was diagnosed with multilineage dysplasia (RCMD) at 14 years. Their mother (I.1, 44 years) remains an asymptomatic carrier, with normal $\mathrm{PB}$ indices and no history of mucosal bleeding or bruising to suggest platelet dysfunction (Figure 1c).

Cytogenetic analysis of tumor samples revealed gain of 21q (46,XX, der(21).ish amp(RUNX1)[15]/46,XX[5]) in II.1, amplifying the germline RUNX1 variant (c.601C > T (p. $\left.\left(\operatorname{Arg} 201^{*}\right)\right)$ WES variant allele frequency (VAF) 67\%); additional events included monosomy 7 and deletion of $9 \mathrm{q}(45, \mathrm{XX},-7[2] / 45, \mathrm{XX}, \mathrm{idem}, \operatorname{del}(9)(\mathrm{q} 21 \mathrm{q} 31)[17] / 46, \mathrm{XX}$ [1]) in II.2, while II.3 and II.4 had a normal karyotype. WES identified a median of 11 somatic non-synonymous variants (range 10-11), with 97\% ( $n=39)$ comprising SNVs, Supplementary Table S3. Targeted analysis of WES data from the mother and father confirmed the absence of variants in established pre-leukemic loci, confirming that additional germline lesions with known pathogenic relevance were not harbored by the siblings.

All three siblings with AML (II.2, II.3 and II.4) acquired variants upregulating JAK2 signaling, as shown in Figure 1c. In II.2 and II.4, JAK2 c.1849G > T (p.(Val617Phe)) was detected, with the variant dosage amplified by $9 \mathrm{p}$ aUPD in II.2 (p.(Val617Phe) VAF 68\%, Figure 2a(i)). Sibling II.1 acquired a unique variant (c.1175G $>$ A (p.(Arg392Gln))) in SH2B3, with 12q aUPD increasing its allelic burden to 67\% (Figure 2a(ii)). SH2B3 (also known as LNK) is a member of the SH2B family of adaptor proteins and negatively regulates JAK2 by interacting with the phosphorylated JAK2 tyrosine residue 813 (p.Tyr813) via a conserved SH2 domain, regulating both WT and mutant JAK2 activity. ${ }^{8}$ By analyzing the structure of murine Sh2b, we identified that SH2B3 p.Arg392 corresponds to Sh2b p.Arg555, ${ }^{9}$ situated in the midst of a precisely conserved region spanning 15 amino acids and forming a salt bridge with the phosphopeptide binding pocket targeted by phosphorylated JAK2. SH2B3 c.1175G $>$ A (p. (Arg392Gln)) is a unique variant, predicted to be damaging using MutationTaster $(P=1.0000,4$ d.p., http://www. mutationtaster.org) and absent from both ExAC (http://exac.broadinstitute.org) and Thousand Genome databases (http://www.internationalgenome.org/data/). We hypothesize that p.(Arg392Gln) may therefore disrupt salt bridging with the phosphopeptide binding pocket in SH2B3, consequently reducing JAK2 inhibition, as previously described with missense variants in this domain. ${ }^{10}$

Additional somatic variants identified across the siblings included the anaphase promoting complex component, CDC27, in II.2 (c.1901T > C (p.(Ile634Thr)), VAF 16.7\%) and II.3 (c.1477A > G (p.(Ile493Val)), VAF 7.5\%); RBBP8 (DNA double-strand break repair, c.959A > G (p.(Glu320Gly)), VAF 32.3\%) and CHD4 (nucleosome remodeling and histone deacetylase complex, c.3530G $>\mathrm{A}$ (p.(Arg1177His)), VAF 5.6\%) in II.2 and U2AF2 (spliceosome component, c.439C > G (p.(Gln147Glu)), VAF 41.2\%) in sibling II.4. These molecular lesions are infrequently observed in sporadic MDS/AML, mirroring observations within other RUNX1 pedigrees where only one to two recurrently mutated driver events are detected in tumors. ${ }^{3,6,7,11}$

Having established the spectrum of variants across the siblings, we next sought to derive the chronological sequence of chromosomal and 

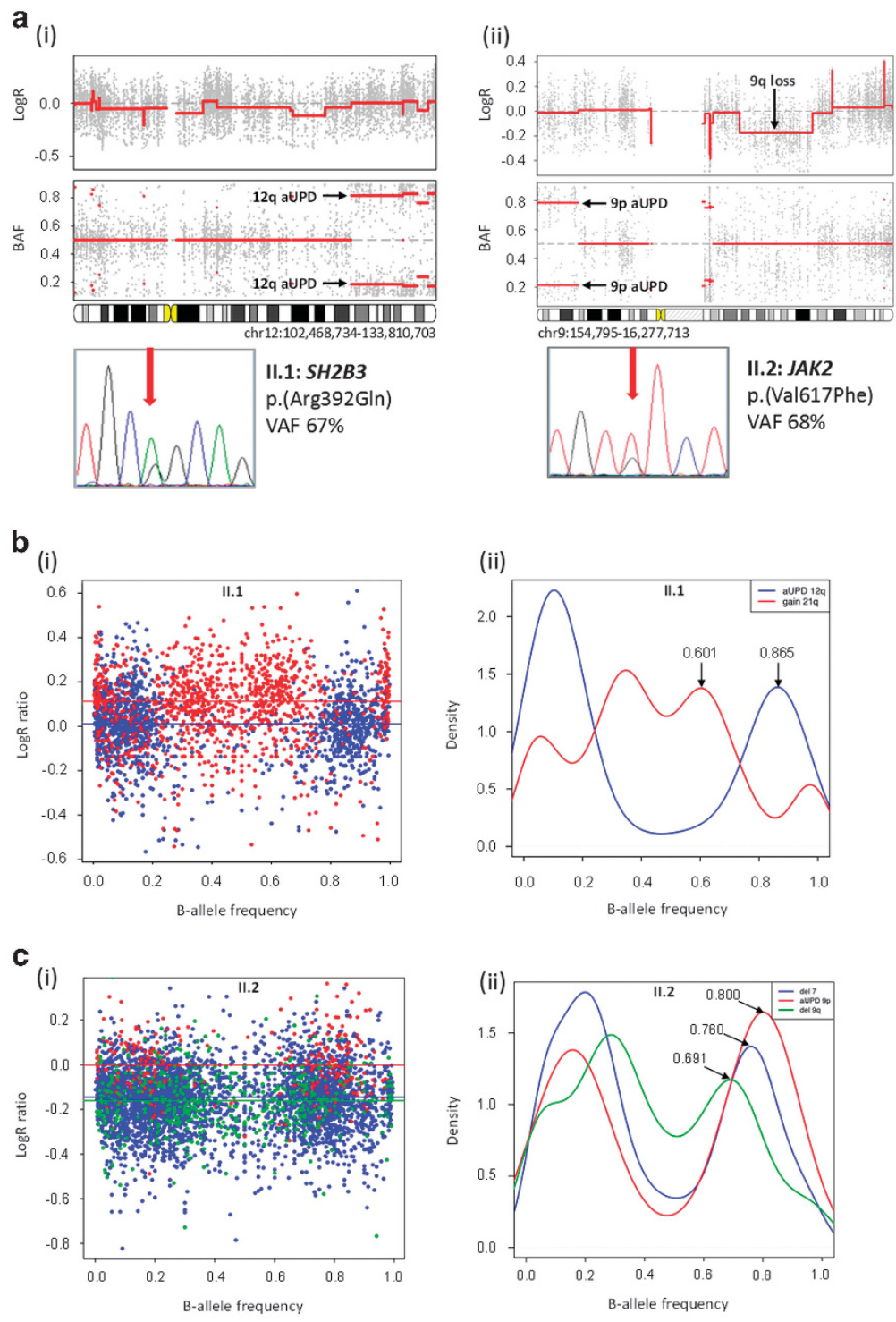

d

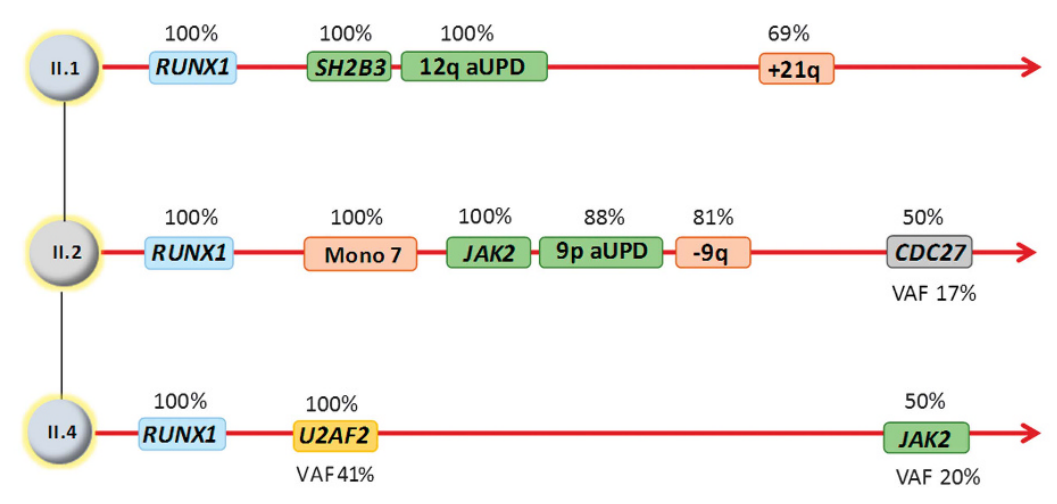

Predisposing event

Co-operating genetic events

genetic aberrations by calculating their respective cancer cell fractions based on the 'Battenberg' algorithm ${ }^{12}$ (Figures $2 \mathrm{~b}$ and $\mathrm{c}$; Supplementary Data). In II.1, SH2B3 c.1175G > A (p.(Arg392Gln)) and 12q aUPD preceded gain of 21q; while in II.2, monosomy 7, JAK2 c.1849G $>$ T (p.(Val617Phe)) and 12q aUPD were followed by $9 q$ deletion (Figure 2d). This pattern of disease evolution revealed the selective and sequential amplification of RUNX1, JAK2 and SH2B3 variants, affirming their key roles as leukemic driver lesions.

The rarity of JAK2 and SH2B3 variants in sporadic AML cases, ${ }^{13}$ suggested these siblings may share an inherited genotype predisposing 
Figure 2 Detailed analysis of chromosomal LOH to decipher sequence of genetic lesions. (a) (i) WES B-allele BAFs demonstrated 12q aUPD (hg19 coordinates: chr12:102,468,734-133,810,703), causing homozygosity of the SH2B3 c.1175G >A (p.(Arg392GIn)) variant in II.1. In II.2, 9p aUPD (hg19 coordinates: chr9:154,795-16,277,713), led to homozygosity of JAK2 c.1849G >T (p.(Val617Phe)). 9q loss was also detected in II.2, characterized by reduced LogR LRR. (b) (i) Plot of LRR and BAF for variants within 12q (blue) and 21q (red) in II.1. (ii) Density plots showing the BAF of variants within regions of LOH, the peak density was used to calculate the CCF of each chromosomal lesion. (c) (i) Plot of LRR and BAF for variants on chromosome 7 (blue), 9p (red) and 9q (green) in II.2. (ii) Density plots showing the BAF of variants within the corresponding chromosomal regions, with the peak density used to calculate CCF. (d) From the calculations in $\mathbf{b}$ and $\mathbf{c}$, the CCF for each LOH segment was calculated allowing the evolutionary sequence to be determined in II.1 and II.2. In II.4, somatic LOH was not detected at AML diagnosis and variant CCFs were estimated using VAFs. BAFs, B-allele frequencies; CCF, cancer cell fractions; LOH, loss of heterozygosity; LRR, LogR ratios.

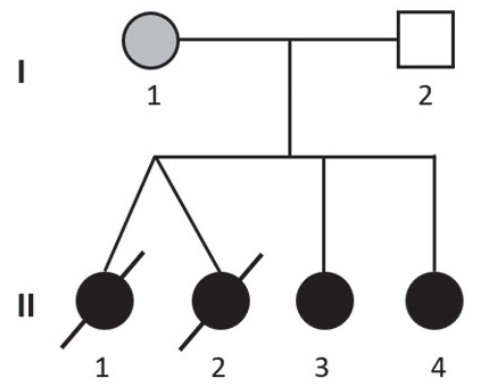

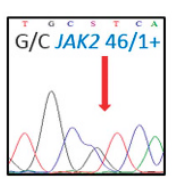

II.1

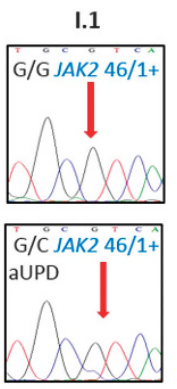

II.2

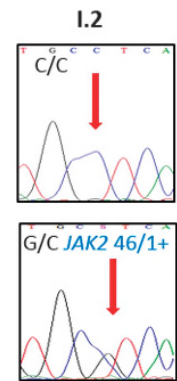

II.3

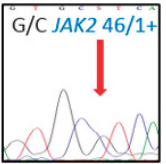

II. 4

Figure 3 Identification of JAK2 46/1 haplotype in mother and children. Sequencing of JAK2 rs12340895 in LD with the 46/1 haplotype, revealed the mother (I.1) was homozygous (GG) for the 46/1 haplotype and the father was homozygous for the non-46/1 allele (CC). All four children were heterozygous for the 46/1 haplotype (GC), with II.2 demonstrating prominence of the 46/1 allele due to 9p aUPD, confirming JAK2 c.1849G > T (p.(Val617Phe)) (also subject to dosage amplification) was acquired on the 46/1 allele. LD, linkage disequilibrium.

to the specific acquisition of JAK-STAT pathway variants. The JAK2 $46 / 1$ haplotype is associated with an increased population risk of developing JAK2 p.(Val617Phe)-positive myeloproliferative neoplasms (MPNs) and sequencing of rs12340895 (in linkage disequilibrium with this haplotype) revealed heterozygosity in all four children (GC). Notably, 9p aUPD in II.2 amplified both V617F and rs12340895 variants, showing that $\mathrm{p}$.(Val617Phe) was acquired on the $46 / 1$ allele, as in sporadic neoplasms. ${ }^{14-16}$ The mother was homozygous for the $46 / 1$ haplotype (GG) and the father was homozygous for the non-46/1 allele (CC) (Figure 3). Subclonal JAK2 and SH2B3 variants were excluded in sibling II.3 and maternal DNA (I.1) by deep sequencing to a depth of $\times 10000$, suggesting that these lesions were specifically acquired upon leukemic transformation, potentially influenced by additional, yet unknown, genetic variants.

All three siblings with AML and JAK2 upregulation demonstrated an aggressive disease course, characterized by relapse and resistance to chemotherapy. Following combination chemotherapy, II.1 achieved complete remission (CR) but died 1 year later with AML relapse. II.2 failed to achieve CR and succumbed to chemotherapy-refractory disease after 8 months. Sibling II.4 received induction chemotherapy with idarubicin, cytarabine and etoposide, and underwent unrelated double cord blood stem cell transplantation (HSCT) following a delay in hematopoietic reconstitution. AML recurred after 17 months requiring a second volunteer unrelated donor HSCT and she continues in remission with full donor chimerism 30 months post transplant.

\section{DISCUSSION}

In this study, we report a novel RUNX1 pedigree with recurrence of somatic JAK-STAT pathway variants in three siblings with aggressive myelomonocytic AML. Our data suggest that inherited genetic traits may govern leukemic transformation and disease behavior by influencing somatic variant acquisition and molecular co-operation. Concurrent disruption of JAK2 and RUNX1 has been reported in sporadic AML, where JAK2 variants occur in $5-8 \%$ of core binding factor leukemia with the $\mathrm{t}(8 ; 21)(\mathrm{q} 22 ; \mathrm{q} 22)$ RUNX1-RUNX1T1 translocation ${ }^{17,18}$ and in a study of 200 AML cases, solitary SH2B3 and JAK2 c.1849G > T (p.(Val617Phe)) variants both coincided with RUNX1 variants. ${ }^{13}$ Conversely, RUNX1 variants have also been reported during leukemic transformation of JAK2-positive MPNs. ${ }^{19,20}$ The lack of recurrent JAK-STAT variants in a recent study of RUNX1-mutated AML ${ }^{1}$ suggests that this co-operation is rare and likely to be governed by additional features, including haplotype. Clinically, all three siblings with somatic JAK-STAT pathway variants demonstrated an adverse disease profile, highlighting the importance of observing phenotypic patterns of disease across family members to improve investigation, management and counseling of these rare pedigrees.

\section{CONFLICT OF INTEREST}

The authors declare no conflict of interest.

\section{ACKNOWLEDGEMENTS}

We are indebted to the family for the opportunity to perform this detailed analysis and to all who collaborated on this project. We would also like to thank $\mathrm{G}$ Clark at the London Research Institute for the automated DNA sequencing work and A Guerra for his help with computational modeling of the SH2B3 variant and A Tordai for providing us with valuable DNA samples. This study was funded by Cancer Research UK (CR-UK) through a Clinical Research Fellowship awarded to KT and a Bloodwise programme grant (14032 to JF). Additional funding was awarded from Children with Cancer (JF), Momentum grant of the Hungarian Academy of Sciences (grant to CB) and the NVKP_161-2016-0004 grant of the Hungarian National Research, Development and Innovation Office (NFKIH).

\section{AUTHOR CONTRIBUTIONS}

KT, JW and CB wrote the paper. KT, JW, JF, JC and CB designed research and provided materials. KT, PK, AAS and AR-M performed research. KT, JW and CC performed data analysis. KK, GB, MZ, AM and JC provided clinical data. 
1 Gaidzik VI, Teleanu V, Papaemmanuil E et al: RUNX1 mutations in acute myeloid leukemia are associated with distinct clinico-pathologic and genetic features. Leukemia 2016; 30: 2282

2 Song WJ, Sullivan MG, Legare RD et al: Haploinsufficiency of CBFA2 causes familial thrombocytopenia with propensity to develop acute myelogenous leukaemia. Nat Genet 1999; 23: 166-175.

3 Antony-Debre I, Duployez N, Bucci M et al: Somatic mutations associated with leukemic progression of familial platelet disorder with predisposition to acute myeloid leukemia. Leukemia 2016; 30: 999-1002.

4 Preudhomme C, Renneville A, Bourdon V et al: High frequency of RUNX1 biallelic alteration in acute myeloid leukemia secondary to familial platelet disorder. Blood 2009; 113: 5583-5587.

5 Yoshimi A, Toya T, Kawazu M et al: Recurrent CDC25C mutations drive malignant transformation in FPD/AML. Nat Commun 2014; 5: 4770.

6 Churpek JE, Pyrtel K, Kanchi KL et al: Genomic analysis of germ line and somatic variants in familial myelodysplasia/acute myeloid leukemia. Blood 2015; 126: 2484-2490.

7 Haslam K, Langabeer SE, Hayat A, Conneally E, Vandenberghe E: Targeted nextgeneration sequencing of familial platelet disorder with predisposition to acute myeloid leukaemia. Br J Haematol 2016; 175: 161-163.

8 Bersenev A, Wu C, Balcerek J, Tong W: Lnk controls mouse hematopoietic stem cell self-renewal and quiescence through direct interactions with JAK2. J Clin Invest 2008; 118: 2832-2844

$9 \mathrm{Hu}$ J, Hubbard SR: Structural basis for phosphotyrosine recognition by the Src homology-2 domains of the adapter proteins SH2-B and APS. J Mol Biol 2006; 361: 69-79.
10 Auer PL, Teumer A, Schick U et al: Rare and low-frequency coding variants in CXCR2 and other genes are associated with hematological traits. Nat Genet 2014; 46: 629-634.

11 Sakurai M, Kasahara H, Yoshida K et al: Genetic basis of myeloid transformation in familial platelet disorder/acute myeloid leukemia patients with haploinsufficient RUNX1 allele. Blood Cancer J 2016; 6: e392.

12 Nik-Zainal S, Van Loo P, Wedge DC et al: The life history of 21 breast cancers. Cell 2012; 149(5): 994-1007.

13 Cancer Genome Atlas Research N: Genomic and epigenomic landscapes of adult de novo acute myeloid leukemia. N Engl J Med 2013; 368: 2059-2074.

14 Jones AV, Chase A, Silver RT et al: JAK2 haplotype is a major risk factor for the development of myeloproliferative neoplasms. Nat Genet 2009; 41: 446-449.

15 Kilpivaara O, Mukherjee S, Schram AM et al: A germline JAK2 SNP is associated with predisposition to the development of JAK2(V617F)-positive myeloproliferative neoplasms. Nat Genet 2009; 41: 455-459.

16 Olcaydu D, Harutyunyan A, Jager R et al: A common JAK2 haplotype confers susceptibility to myeloproliferative neoplasms. Nat Genet 2009; 41: 450-454.

17 Krauth MT, Eder C, Alpermann T et al: High number of additional genetic lesions in acute myeloid leukemia with $\mathrm{t}(8 ; 21) / R U N X 1-R U N X 1 T 1$ : frequency and impact on clinical outcome. Leukemia 2014; 28: 1449-1458.

18 Schnittger S, Bacher U, Kern W, Haferlach C, Haferlach T: JAK2 seems to be a typical cooperating mutation in therapy-related $\mathrm{t}(8 ; 21) / \mathrm{AML1}-\mathrm{ETO}$-positive AML. Leukemia 2007; 21: 183-184.

19 Beer PA, Delhommeau F, LeCouedic JP et al: Two routes to leukemic transformation after a JAK2 mutation-positive myeloproliferative neoplasm. Blood 2010; 115: 2891-2900.

20 Engle EK, Fisher DA, Miller CA et al: Clonal evolution revealed by whole genome sequencing in a case of primary myelofibrosis transformed to secondary acute myeloid leukemia. Leukemia 2015; 29: 869-876.

Supplementary Information accompanies this paper on European Journal of Human Genetics website (http://www.nature.com/ejhg) 